\title{
Alternative cleavage and polyadenylation sites in colorectal cancer
}

\author{
Y. Kang ${ }^{1 *}$, X. Yang ${ }^{1}$, W. Xu ${ }^{1}$, Z. Mao ${ }^{1}$, J. Sun ${ }^{2}$ \\ ${ }^{1}$ School of Biomedical Engineering, Bio-ID Center, Shanghai Jiao Tong University, Shanghai, China \\ ${ }^{2}$ Shanghai Center for Systems Biomedicine, Shanghai Jiao Tong University, Shanghai, China \\ *e-mail:kangyani@sjtu.edu.cn
}

Key words: alternative polyadenylation, colorectal cancer, 3T-seq, 3'UTR

Motivation and Aim: Alternative polyadenylation (APA) is an important post-transcriptional regulation in eukaryotic cells. It plays considerable roles in many biological processes and diseases, such as cell differentiation, proliferation and cancer. Colorectal cancer is one of the most common malignancies worldwide, which is among the top five in incidence and mortality of all cancers in China, even though China is not a high prevalence area. Studies have shown that $\sim 70 \%$ of protein-coding genes have conserved microRNA (miRNA) target sites within their 3'UTRs. Moreover, during transformation, the cell starts using the poly (A) sites (PAS) most proximal to the open reading frame (ORF) to generate a short 3'UTR, which makes the mRNA resistant to miRNA by eliminating miRNA-binding sites. Recent studies reported a widespread preferential usage of proximal PAS in cancers, such as breast, lung, liver, and colorectal cancers. Even if, the role of APA in transformation and cancer is still not very clear. Although there have been some studies on the APA of colorectal cancer, the normal and carcinoma samples used for genome-wide profiling were not matched. The purpose of this study was to obtain genes with switched 3' untranslated region (UTR) that may be associated with intracellular regulation of colorectal cancer by analyzing APA patterns of strict control groups from clinical patients.

Results: We used a robust approach, 3T-seq to profile global APA sites in three patients and observed hundreds genes exhibit shortened 3'UTR, and some of them have been reported play a key role in cancer. Overall, we identified 35,076 poly (A) sites in total. Compared to the matched normal tissues, we detected 350, 405 and 375 genes with significantly APA-mediated 3'UTR alteration in cancer tissues of 3 patients, respectively. Forty-seven genes with switched 3'UTR were shared in all three patients. In addition, most of these genes have shortened 3'UTRs, some of which were associated with cancers, such as GPI.

Conclusion: Our studies found several genes with switched 3'UTR in colorectal cancer patients, which may provide some important clues for more in-depth study of the cellular regulation in colorectal cancer from the perspective of post-transcriptional regulation. It may also help in the search for new biomarkers of colorectal cancers.

Acknowledgements: This work was supported by National Natural Science Foundation of China (31671299), Meng Minwei foundation, Medical engineering cross fund (YG2017ZD15, YG2015QN35) and Laboratory Innovative Research Program of Shanghai Jiao Tong University (17SJ-18). 